\title{
Variants in the GH-IGF axis confer susceptibility to lung cancer
}

\author{
Matthew F. Rudd, ${ }^{1}$ Emily L. Webb, ${ }^{1}$ Athena Matakidou, ${ }^{1}$ Gabrielle S. Sellick, ${ }^{1}$ \\ Richard D. Williams, ${ }^{2}$ Helen Bridle, ${ }^{3}$ Tim Eisen, ${ }^{3,4}$ Richard S. Houlston, ${ }^{1,4,5}$ \\ and the GELCAPS Consortium ${ }^{6}$ \\ ${ }^{1}$ Section of Cancer Genetics, ${ }^{2}$ Section of Paediatrics, and ${ }^{3}$ Section of Medicine, Institute of Cancer Research, Sutton, \\ Surrey SM2 5NG, United Kingdom
}

\begin{abstract}
We conducted a large-scale genome-wide association study in UK Caucasians to identify susceptibility alleles for lung cancer, analyzing 1529 cases and 2707 controls. To increase the likelihood of identifying disease-causing alleles, we genotyped 1476 nonsynonymous single nucleotide polymorphisms (nsSNPs) in 871 candidate cancer genes, biasing SNP selection toward those predicted to be deleterious. Statistically significant associations were identified for 64 nsSNPs, generating a genome-wide significance level of $P=0.002$. Eleven of the 64 SNPs mapped to genes encoding pivotal components of the growth hormone/insulin-like growth factor (GH-IGF) pathway, including CAMKKI E375G $\left(\mathrm{OR}=1.37, P=5.4 \times 10^{-5}\right)$, AKAP9 M463I $\left(\mathrm{OR}=1.32, P=1.0 \times 10^{-4}\right)$ and $G H R$ P495T $(\mathrm{OR}=12.98, P=0.0019)$. Significant associations were also detected for SNPs within genes in the DNA damage-response pathway, including BRCA2 K3326X (OR $=1.72, P=0.0075)$ and XRCC4 II37T (OR $=1.31, P=0.0205)$. Our study provides evidence that inherited predisposition to lung cancer is in part mediated through low-penetrance alleles and specifically identifies variants in GH-IGF and DNA damage-response pathways with risk of lung cancer.
\end{abstract}

[Supplemental material is available online at www.genome.org.]

Lung cancer is the most common cancer in the world and represents a major public health problem, accounting for $\sim 1.2$ million cancer-related deaths worldwide each year (Parkin et al. 2005). Tobacco smoking is acknowledged to be the major risk factor for lung cancer, contributing to a 10 -fold increase in risk in long-term smokers compared with nonsmokers (Doll and Peto 1981). Other environmental risk factors include exposure to radiation, asbestos, heavy metals, polycyclic aromatic hydrocarbons, and chloromethyl ethers (IARC 1986).

Lung cancer is frequently cited as a malignancy solely attributable to environmental exposure. However, it has long been postulated that individuals may differ in their susceptibility and there is increasing evidence from epidemiological studies for a familial risk (Matakidou et al. 2005). Direct evidence for a genetic predisposition is provided by the increased risk of lung cancer associated with a number of rare Mendelian cancer syndromes, such as carriers of constitutional tumor protein p53 (TP53) (Hwang et al. 2003) and retinoblastoma (Sanders et al. 1989) gene mutations, as well as in patients with Bloom's (Takemiya et al. 1987) and Werner's syndromes (Yamanaka et al. 1997).

The genetic basis of inherited susceptibility to lung cancer outside the context of the rare Mendelian cancer predisposition syndromes is at present undefined, but a model in which dominantly acting, high-risk alleles account for all of the excess familial risk seems unlikely. An alternative hypothesis about the allelic architecture of lung cancer susceptibility proposes that most of the genetic risk is caused by low-penetrance alleles. This hypoth-

\footnotetext{
${ }^{4}$ These two authors contributed equally to this work. ${ }^{5}$ Corresponding author.

E-mail Richard.Houlston@icr.ac.uk; fax 4-20-8722-4359.

${ }^{6}$ List of GELCAPS Consortium collaborators available on request.

Article is online at http://www.genome.org/cgi/doi/10.1101/gr.5120106.
}

esis implies that testing for allelic association should be a powerful strategy for identifying lung cancer predisposition alleles.

We sought to identify novel low-penetrance susceptibility alleles to lung cancer by genotyping SNPs across 871 genes with relevance to cancer biology. To increase the likelihood of identifying disease-causing alleles, we biased selection of nsSNPs to those likely to have functionally deleterious consequences. Genotyping 1529 lung cancer cases and 2707 controls from the UK population across 1476 nsSNPs provided strong evidence that low-penetrance alleles in genes involved in the hormone/insulinlike growth factor $(G H-I G F)$ and DNA damage-response pathways are associated with lung cancer susceptibility

\section{Results}

Genotypes were obtained for 1526 cases (99.8\%) and 2695 controls (99.6\%). Of the 1476 SNPs submitted for analysis, 1221 SNPs had sample call rates $>95 \%$. Of these, 180 were fixed, leaving 1041 SNPs for which genotype data were informative (Supplemental Table 1). Implementing the genomic control method indicated no evidence of population stratification in our data as a cause of false-positive results, as the $95 \%$ confidence interval for the stratification parameter $\hat{\lambda}(0.92-1.31)$ encompassed unity. As deviates from Hardy-Weinberg equilibrium followed the expected distribution, we concluded that genotyping error is unlikely to have impacted on the statistics generated.

Significant associations with risk of lung cancer were identified for 64 of 1041 nsSNPs at the 5\% level. The overrepresentation of associations between SNPs and lung cancer risk was confirmed by a joint analysis of their combined effect using the set-association approach (smallest global significance level of $P=4.2 \times 10^{-4}$ ). After further adjustment for the number of 
terms in the set being a priori, unknown, the genome-wide significance was $P=0.002$.

Two of the 64 SNPs identified through the set association procedure, rs2602141 (K1136Q) and rs560191 (D353E), map to the tumor protein p53 binding protein 1 (TP53BP1) and are in strong linkage disequilibrium (LD). A further group of three SNPs in the MHC region spaced within $100 \mathrm{~kb}$; rs1052486 (S625P) in HLA-B-associated transcript 3 (BAT3), rs3130618 (R41L) in HLAB-associated transcript 4 (BAT4), and rs16900023 (P786S) in mutS homolog 5 (MSH5) also formed a cluster of high LD. Although the permutation procedure implemented in the setassociation strategy allows for such substructure in the data when estimating significance levels, it may not be desirable to include highly correlated SNPs in the analysis. A total of 262 SNPs displayed high LD with an adjacent SNP. High LD was found to occur primarily within the same gene, but there were 80 instances where strong LD was observed between SNPs in different genes. We repeated our analysis by omitting markers in $\mathrm{LD}$, retaining one SNP per LD set on the basis of maximum GenCall score or call rate, yielding almost identical sum statistics $(P=0.005)$ with inclusion of 70 SNPs.

Sixty-seven SNPs displayed significant association at the 5\% level with familial lung cancer, but only 52 when the analysis was restricted to sporadic cases. After permutation, the overall significance level attained from the set-association analysis for the familial cases was $P=0.015$ compared with $P=0.076$ for sporadic cases. Familial cases contributed significantly to overall study findings with 13 SNPs contributing to the 20 associated at the $1 \%$ level in the overall data set (Table 1). Stratification of cases by cancer histology (small cell and non-small cell, global $P$-values 0.11 and 0.06 , respectively), age at diagnosis $(<60$ and $\geq 60$; global $P$-values 0.17 and 0.18 , respectively) and sex (male and female; global $P$-values 0.19 and 0.08 , respectively) did not impact significantly on study findings. Furthermore, limiting our analysis to the $93.7 \%$ of cases who were smokers indicated that there was no evidence of confounding due to smoking.

The SNP showing the most significant allelic association with lung cancer was rs1052486 (S625P) in BAT3, a nuclear protein implicated in the control of apoptosis, with strongest association under a recessive model $\left(\mathrm{OR}_{\mathrm{R}}=0.69,95 \%\right.$ CI: 0.59-0.82, $\left.P_{\mathrm{R}}=8.3 \times 10^{-6}\right)($ Table 1$)$. Two additional SNPs, rs7214723 (E375G) in calcium/calmodulin-dependent protein kinase kinase $1 \alpha(C A M K K 1)$, belonging to the Serine/Threonine protein kinase family $\left(\mathrm{OR}_{\mathrm{R}}=1.37,95 \% \mathrm{CI}: 1.17-1.59, P_{\mathrm{R}}=5.4 \times 10^{-5}\right)$ and rs6964587 (M463I) in A kinase anchor protein 9 (AKAP9), a key component of signal transduction $\left(\mathrm{OR}_{\mathrm{D}}=1.32\right.$, 95\% CI: $1.15-$ $1.52, P_{\mathrm{D}}=7.6 \times 10^{-5}$ ), also showed highly significant nominal association under recessive and dominant models, respectively. Empirical limits for genome-wide significance for individual $\mathrm{T}_{\mathrm{A}}$, $\mathrm{T}_{\mathrm{D}}$, and $\mathrm{T}_{\mathrm{R}}$ statistics were established at 16.12, 16.23, and 15.66, respectively. Hence, BAT3 S625P and CAMKK1 E375G were both significantly associated with lung cancer with adjusted $P$-values of 0.006 and 0.036 , respectively, with AKAP9 M463I showing borderline significance with adjusted $P=0.066$.

Of the 64 SNPs identified, two SNPs have been documented to be functional, i.e., K3326X in breast cancer 2 early onset (BRCA2) and N700S in thrombospondin 1 (THBS1), and a further 37 SNPs are predicted in silico to deleteriously impact on the expressed proteins (Table 2).

Through interrogation of the Pathway Assist program (Stratagene), 11 of the 64 SNPs associated with risk of lung cancer were located within individual genes encoding pivotal compo- nents of the extended GH-IGF pathway, including CAMKK1 E375G and AKAP9 M463I (both of which were globally significant), growth hormone receptor $(G H R)$ P495T $\left(\mathrm{OR}_{\mathrm{D}}=12.98\right.$, $\left.P_{\mathrm{D}}=0.0019\right)$, A kinase anchor protein 10 (AKAP10) $\mathrm{R} 249 \mathrm{H}$ $\left(\mathrm{OR}_{\mathrm{R}}=1.25, P_{\mathrm{R}}=0.0085\right)$, and insulin-like growth-factor binding protein 5 (IGFBP5) R138W $\left(\mathrm{OR}_{\mathrm{D}}=1.29, P_{\mathrm{D}}=0.027\right)$ (Table 1$)$. A further five SNPs were located in genes directly involved in the DNA damage-response pathway, including the functional BRCA2 SNP K3326X $\left(\mathrm{OR}_{\mathrm{D}}=1.72, P_{\mathrm{D}}=0.0075\right)$, X-ray repair complementing defective repair in Chinese hamster cells 4 (XRCC4) I134T $\left(\mathrm{OR}_{\mathrm{D}}=1.31, P_{\mathrm{D}}=0.0205\right)$, mutS homolog 5 (MSH5) P786S $\left(\mathrm{OR}_{\mathrm{D}}=0.64, P_{\mathrm{D}}=0.0228\right)$, mutS homolog $4(\mathrm{MSH} 4) \mathrm{S} 914 \mathrm{~N}$ $\left(\mathrm{OR}_{\mathrm{D}}=1.27, P_{\mathrm{D}}=0.0461\right)$, and BRCA1-associated RING domain 1 (BARD1) R658C $\left(\mathrm{OR}_{\mathrm{D}}=1.59, P_{\mathrm{D}}=0.0329\right)$ (Table 1$)$.

Haplotype frequencies defined by the two sets of SNPs displaying high LD, TP53BP1 K1136Q and D353E, and BAT3 S625P, BAT4 R41L, and MSH5 P786S were significantly different in cases and controls (adjusted $P$-values, 0.01 and 0.01 , respectively, after permutation testing).

We examined for potential interactive effects between the 64 SNPs significantly associated with lung cancer risk $\left(P_{\mathrm{A}}<0.05\right)$ by fitting full logistic regression models for each pair, generating 2016 models, and comparing these with the main effects model. Ninety-six pairs of SNPs showed nominally significant interaction at the 5\% level. The largest interactive effect identified was between 1-aminocyclopropane-1-carboxylate synthase (PHACS) P421L and toll-like receptor 1 (TLR1) R80T $\left(P=3.3 \times 10^{-4}\right)$, albeit nonsignificant after correction for multiple testing.

\section{Discussion}

To date, the only evidence for a major locus for lung cancer susceptibility is provided by the linkage scan conducted by Bailey-Wilson et al. (2004), which reported linkage of the disease to chromosome 6q23-25, and a model based on involvement of multiple low-penetrance alleles is eminently plausible.

Previous association studies aimed at identifying lowpenetrance alleles for lung cancer susceptibility have evaluated a restricted number of polymorphisms, primarily in genes implicated in the metabolism of tobacco-associated carcinogens and protection of DNA from carcinogen-induced damage. To identify novel lung cancer susceptibility alleles, we extended our search to include genes with relevance to cancer biology, evaluating only nsSNPs that have a higher probability of being directly causal. We acknowledge that the loci considered as candidates will be based on current preconceptions of cancer biology, and it is likely that other genes may influence tumor development. The number of candidate loci will inevitably increase with advances in cancer biology.

The number of nsSNPs that displayed significant association with lung cancer risk was greater than that expected, supporting the tenet that polymorphic variation contributes to lung cancer susceptibility. This assertion is supported by the fact that associations were stronger when the analysis was restricted to those cases with a family history of lung cancer. We cannot exclude the possibility that some of the associations detected are a consequence of LD with causal mutations. It is noteworthy that the SNPs in BAT3, BAT4, and MSH5, which were all associated with lung cancer risk, were in strong LD.

Of the 64 SNPs found to be associated with lung cancer risk, several reside in genes involved in either apoptosis (BARD1 and 
Functional nsSNPs and lung cancer risk

Table 1. SNPs showing significant allelic association with lung cancer

\begin{tabular}{|c|c|c|c|c|c|c|c|}
\hline \multirow[b]{2}{*}{ SNP } & \multirow[b]{2}{*}{ Gene $^{a}$} & \multirow[b]{2}{*}{ Substitution } & \multirow[b]{2}{*}{$\mathrm{MAF}^{\mathrm{b}}$} & \multicolumn{2}{|c|}{ Allelic statistic } & \multicolumn{2}{|c|}{ Dominant/Recessive statistics } \\
\hline & & & & OR $(95 \% \mathrm{Cl})$ & $P_{\mathrm{A}}$ & $\mathrm{OR}_{\mathrm{D} / \mathrm{R}}(95 \% \mathrm{Cl})^{\mathrm{c}}$ & $P_{\mathrm{D} / \mathrm{R}}^{\mathrm{c}}$ \\
\hline rs $1052486^{d}$ & BAT3 & S625P & 0.483 & $0.84(0.77,0.92)$ & 0.0002 & $0.69(0.59,0.82)^{R}$ & $8.3 \times 10^{-6 R}$ \\
\hline rs $7214723^{d}$ & CAMKK1 & E375G & 0.446 & $1.18(1.08,1.29)$ & 0.0003 & $1.37(1.17,1.59)^{R}$ & $5.4 \times 10^{-5 R}$ \\
\hline $\operatorname{rs} 560191^{d}$ & TP53BP1 & D353E & 0.305 & $0.85(0.77,0.93)$ & 0.0009 & $0.84(0.74,0.95)^{\mathrm{D}}$ & $0.0050^{\mathrm{D}}$ \\
\hline rs $3130618^{d}$ & BAT4 & R41L & 0.190 & $1.20(1.07,1.34)$ & 0.0013 & $1.26(1.11,1.44)^{\mathrm{D}}$ & $0.0005^{\mathrm{D}}$ \\
\hline rs $2602141^{d}$ & TP5 3BP1 & K1136Q & 0.304 & $0.85(0.77,0.94)$ & 0.0014 & $0.84(0.74,0.95)^{\mathrm{D}}$ & $0.0063^{\mathrm{D}}$ \\
\hline rs6964587 d & AKAPG & M463I & 0.383 & $1.16(1.06,1.28)$ & 0.0016 & $1.32(1.15,1.52)^{\mathrm{D}}$ & $0.0001^{\mathrm{D}}$ \\
\hline rs2229742 & NRIP1 & R448G & 0.101 & $1.25(1.08,1.43)$ & 0.0021 & $1.24(1.07,1.45)^{\mathrm{D}}$ & $0.0052^{\mathrm{D}}$ \\
\hline rs2660744 & PPAT & Q488X & 0.155 & $0.82(0.72,0.93)$ & 0.0024 & $0.80(0.69,0.93)^{\mathrm{D}}$ & $0.0026^{\mathrm{D}}$ \\
\hline rs3206824 & DKK3 & R335G & 0.231 & $1.16(1.05,1.29)$ & 0.0046 & $1.21(1.07,1.38)^{\mathrm{D}}$ & $0.0028^{\mathrm{D}}$ \\
\hline rs6183 & GHR & P495T & 0.001 & $12.98(1.77, \infty)$ & 0.0047 & $12.98(1.77, \infty)^{\mathrm{D}^{\prime}}$ & $0.0019^{\mathrm{D}}$ \\
\hline rs1129923 & DUSP23 & G131S & 0.097 & $0.80(0.68,0.93)$ & 0.0050 & $0.79(0.67,0.94)^{\mathrm{D}}$ & $0.0069^{\mathrm{D}}$ \\
\hline rs $11571833^{d}$ & BRCA2 & K3326X & 0.009 & $1.74(1.17,2.59)$ & 0.0054 & $1.72(1.15,2.57)^{\mathrm{D}}$ & $0.0075^{\mathrm{D}}$ \\
\hline rs 2242089 & PYCRL & V105M & 0.183 & $0.84(0.75,0.95)$ & 0.0054 & $0.81(0.71,0.93)^{\mathrm{D}}$ & $0.0028^{\mathrm{D}}$ \\
\hline rs $1738023^{d}$ & $A K R 7 A 3$ & N215D & 0.164 & $1.18(1.05,1.32)$ & 0.0063 & $1.19(1.04,1.37)^{\mathrm{D}}$ & $0.0098^{\mathrm{D}}$ \\
\hline rs11569705 & SULT1E1 & $\mathrm{D} 22 \mathrm{Y}$ & 0.003 & $0.10(0.01,0.78)$ & 0.0068 & $0.10(0.01,0.78)^{\mathrm{D}}$ & $0.0068^{\mathrm{D}}$ \\
\hline rs $2295778^{d}$ & HIFTAN & P41A & 0.263 & $1.15(1.04,1.26)$ & 0.0070 & $1.34(1.07,1.69)^{R}$ & $0.0112^{R}$ \\
\hline rs2306022 & ITGA11 & V433M & 0.094 & $0.80(0.68,0.94)$ & 0.0072 & $0.79(0.66,0.94)^{\mathrm{D}}$ & $0.0065^{\mathrm{D}}$ \\
\hline rs10115703 & CER1 & R19W & 0.079 & $0.79(0.66,0.94)$ & 0.0088 & $0.76(0.63,0.92)^{\mathrm{D}}$ & $0.0041^{\mathrm{D}}$ \\
\hline rs $2108978^{d}$ & AKAP10 & $\mathrm{R} 249 \mathrm{H}$ & 0.393 & $1.13(1.03,1.23)$ & 0.0092 & $1.25(1.06,1.48)^{R}$ & $0.0085^{R}$ \\
\hline rs $2725362^{d}$ & WRN & L1074F & 0.473 & $0.89(0.81,0.97)$ & 0.0100 & $0.81(0.69,0.94)^{R}$ & $0.0069^{R}$ \\
\hline rs $3732401^{d}$ & GTF2E1 & P366S & 0.043 & $0.73(0.57,0.93)$ & 0.0112 & $0.72(0.57,0.93)^{\mathrm{D}}$ & $0.0107^{\mathrm{D}}$ \\
\hline rs $4371716^{d}$ & $\mathrm{CDH} 12$ & V68M & 0.242 & $1.14(1.03,1.26)$ & 0.0122 & $1.63(1.27,2.09)^{R}$ & $0.0001^{R}$ \\
\hline rs $8065506^{d}$ & ZNF624 & K135N & 0.265 & $1.13(1.03,1.25)$ & 0.0133 & $1.14(1.01,1.30)^{\mathrm{D}}$ & $0.0355^{\mathrm{D}}$ \\
\hline rs $970547^{d}$ & COL12A1 & G1894S & 0.223 & $0.87(0.78,0.97)$ & 0.0141 & $0.67(0.49,0.93)^{R}$ & $0.0173^{R}$ \\
\hline rs2032729 & ZNF24 & N220S & 0.076 & $1.22(1.04,1.43)$ & 0.0143 & $1.24(1.05,1.47)^{\mathrm{D}}$ & $0.0124^{\mathrm{D}}$ \\
\hline rs2243639 & SFTPD & T180A & 0.408 & $0.89(0.82,0.98)$ & 0.0151 & $0.87(0.76,0.99)^{\mathrm{D}}$ & $0.0330^{\mathrm{D}}$ \\
\hline rs $17632786^{d}$ & THBS1 & N700S & 0.133 & $0.85(0.74,0.97)$ & 0.0163 & $0.81(0.70,0.94)^{\mathrm{D}}$ & $0.0056^{\mathrm{D}}$ \\
\hline rs 10787428 & GPAM & E131G & 0.396 & $0.89(0.82,0.98)$ & 0.0168 & $0.87(0.77,0.99)^{\mathrm{D}}$ & $0.0350^{\mathrm{D}}$ \\
\hline rs17184326 & POP1 & K522N & 0.128 & $1.17(1.03,1.33)$ & 0.0174 & $1.18(1.02,1.36)^{\mathrm{D}}$ & $0.0257^{\mathrm{D}}$ \\
\hline rs11575194 ${ }^{d}$ & IGFBP5 & R138W & 0.038 & $1.29(1.04,1.61)$ & 0.0183 & $1.29(1.03,1.61)^{\mathrm{D}}$ & $0.0270^{\mathrm{D}}$ \\
\hline rs363504 & GRIK1 & L902S & 0.051 & $0.77(0.62,0.96)$ & 0.0194 & $0.15(0.02,1.13)^{R}$ & $0.0324^{R}$ \\
\hline rs 28360135 & $X R C C 4$ & I134T & 0.035 & $1.31(1.04,1.64)$ & 0.0202 & $1.31(1.04,1.66)^{\mathrm{D}}$ & $0.0205^{\mathrm{D}}$ \\
\hline rs $1051740^{d}$ & EPHX1 & Y113H & 0.286 & $1.12(1.02,1.24)$ & 0.0204 & $1.15(1.01,1.30)^{\mathrm{D}}$ & $0.0297^{\mathrm{D}}$ \\
\hline rs $16900023^{d}$ & MSH5 & P786S & 0.018 & $0.64(0.43,0.94)$ & 0.0206 & $0.64(0.43,0.94)^{\mathrm{D}}$ & $0.0228^{\mathrm{D}}$ \\
\hline rs1043261 & $I L 17 R B$ & Q484X & 0.084 & $0.82(0.69,0.97)$ & 0.0222 & $0.82(0.69,0.99)^{\mathrm{D}}$ & $0.0334^{\mathrm{D}}$ \\
\hline rs $2295000^{d}$ & DATF1 & S535L & 0.218 & $0.88(0.79,0.98)$ & 0.0224 & $0.86(0.75,0.98)^{\mathrm{D}}$ & $0.0202^{\mathrm{D}}$ \\
\hline rs1019670 & $M S 4 A 6 A$ & N150I & 0.393 & $0.90(0.82,0.99)$ & 0.0243 & $0.88(0.77,1.00)^{\mathrm{D}}$ & $0.0455^{\mathrm{D}}$ \\
\hline rs7998427 & SETDB2 & E117G & 0.324 & $0.90(0.81,0.99)$ & 0.0248 & $0.87(0.77,0.99)^{\mathrm{D}}$ & $0.0367^{\mathrm{D}}$ \\
\hline rs9262138 & DHX16 & D566G & 0.061 & $0.80(0.65,0.97)$ & 0.0249 & $0.79(0.64,0.97)^{\mathrm{D}}$ & $0.0230^{\mathrm{D}}$ \\
\hline rs5745549 & MSH4 & S914N & 0.033 & $1.30(1.03,1.63)$ & 0.0256 & $1.27(1.00,1.61)^{\mathrm{D}}$ & $0.0461^{\mathrm{D}}$ \\
\hline rs3107275 & PHACS & P421L & 0.406 & $0.90(0.82,0.99)$ & 0.0272 & $0.85(0.71,1.01)^{R}$ & $0.0609^{R}$ \\
\hline rs8069344 & GUCY2D & L782H & 0.135 & $0.86(0.75,0.98)$ & 0.0277 & $0.82(0.70,0.95)^{\mathrm{D}}$ & $0.0078^{\mathrm{D}}$ \\
\hline rs5388 & $\mathrm{GH} 1$ & V136I & 0.012 & $0.59(0.36,0.95)$ & 0.0287 & $0.56(0.34,0.91)^{\mathrm{D}}$ & $0.0185^{\mathrm{D}}$ \\
\hline rs1800974 & ITGA7 & $\mathrm{R} 651 \mathrm{H}$ & 0.488 & $1.10(1.01,1.21)$ & 0.0307 & $1.14(0.98,1.31)^{\mathrm{R}}$ & $0.0803^{R}$ \\
\hline rs 1820128 & ZNF600 & C209R & 0.143 & $0.87(0.76,0.99)$ & 0.0325 & $0.85(0.74,0.98)^{\mathrm{D}}$ & $0.0294^{\mathrm{D}}$ \\
\hline rs17246389 & SERPINI2 & L6V & 0.266 & $0.89(0.81,0.99)$ & 0.0328 & $0.86(0.76,0.98)^{\mathrm{D}}$ & $0.0227^{\mathrm{D}}$ \\
\hline rs17337252 & RB1CC1 & M234T & 0.491 & $1.10(1.01,1.20)$ & 0.0329 & $1.15(1.00,1.33)^{R}$ & $0.0584^{R}$ \\
\hline rs3758938 & $T B \times 10$ & K101T & 0.312 & $0.90(0.82,0.99)$ & 0.0338 & $0.88(0.78,1.00)^{\mathrm{D}}$ & $0.0551^{\mathrm{D}}$ \\
\hline rs 1800076 & CFTR & R75Q & 0.037 & $1.27(1.02,1.58)$ & 0.0339 & $1.27(1.01,1.59)^{\mathrm{D}}$ & $0.0412^{\mathrm{D}}$ \\
\hline rs $2230674^{d}$ & ATF1 & P191A & 0.041 & $0.77(0.60,0.98)$ & 0.0348 & $0.77(0.60,0.99)^{\mathrm{D}}$ & $0.0425^{\mathrm{D}}$ \\
\hline rs 2274750 & TNC & A1781T & 0.025 & $1.32(1.01,1.71)$ & 0.0391 & $1.32(1.01,1.73)^{\mathrm{D}}$ & $0.0391^{\mathrm{D}}$ \\
\hline rs933135 & PLCD1 & R257H & 0.013 & $1.45(1.02,2.06)$ & 0.0391 & $1.48(1.03,2.11)^{\mathrm{D}}$ & $0.0312^{\mathrm{D}}$ \\
\hline rs5743611 & TLR1 & R80T & 0.087 & $0.84(0.71,0.99)$ & 0.0394 & $0.84(0.70,1.00)^{\mathrm{D}}$ & $0.0525^{\mathrm{D}}$ \\
\hline rs1211554 & HUS1B & D268Y & 0.089 & $0.84(0.72,0.99)$ & 0.0415 & $0.85(0.72,1.01)^{\mathrm{D}}$ & $0.0694^{\mathrm{D}}$ \\
\hline rs4647932 & FGFRL1 & P464L & 0.061 & $1.20(1.01,1.43)$ & 0.0415 & $1.19(0.99,1.44)^{\mathrm{D}}$ & $0.0652^{\mathrm{D}}$ \\
\hline rs 17356233 & CHD1L & $\mathrm{H} 350 \mathrm{Q}$ & 0.247 & $0.90(0.81,1.00)$ & 0.0417 & $0.86(0.76,0.98)^{\mathrm{D}}$ & $0.0220^{\mathrm{D}}$ \\
\hline rs $3738888^{d}$ & BARD1 & R658C & 0.009 & $1.55(1.01,2.36)$ & 0.0423 & $1.59(1.03,2.44)^{\mathrm{D}}$ & $0.0329^{\mathrm{D}}$ \\
\hline rs12500797 & PTPN13 & E1606K & 0.107 & $1.15(1.00,1.32)$ & 0.0443 & $1.18(1.01,1.37)^{\mathrm{D}}$ & $0.0364^{\mathrm{D}}$ \\
\hline rs4988492 & $\mathrm{GHRH}$ & L75F & 0.013 & $1.44(1.00,2.05)$ & 0.0470 & $1.44(1.01,2.07)^{\mathrm{D}}$ & $0.0453^{\mathrm{D}}$ \\
\hline rs2230339 & GPR68 & R63Q & 0.001 & $6.80(0.73, \infty)$ & 0.0472 & $6.80(0.73, \infty)^{\mathrm{D}}$ & $0.0213^{\mathrm{D}}$ \\
\hline rs2229424 & FASN & R1694H & 0.001 & $6.80(0.73, \infty)$ & 0.0474 & $6.79(0.73, \infty)^{\mathrm{D}}$ & $0.0214^{\mathrm{D}}$ \\
\hline rs4791641 & PFAS & P367L & 0.497 & $0.91(0.84,1.00)$ & 0.0475 & $0.87(0.75,1.01)^{\mathrm{R}}$ & $0.0668^{R}$ \\
\hline rs11652709 & $E P X$ & Q122H & 0.320 & $1.10(1.00,1.21)$ & 0.0477 & $1.13(0.99,1.28)^{\mathrm{D}}$ & $0.0668^{\mathrm{D}}$ \\
\hline rs 1801690 & $\mathrm{APOH}$ & W335s & 0.056 & $0.81(0.66,1.00)$ & 0.0480 & $0.81(0.66,1.00)^{\mathrm{D}}$ & $0.0549^{\mathrm{D}}$ \\
\hline
\end{tabular}

\footnotetext{
aNCBI Entrez Gene.
}

${ }^{\mathrm{b}}$ Minor allele frequency (MAF) in cases.

cMost significant association under a dominant (D) or recessive (R) model.

${ }^{\mathrm{d}}$ Associated at significance level $5 \%$ when analysis restricted to familial cases. 
Table 2. Description and predicted functionality of nsSNPs showing significant association with lung cancer risk

\begin{tabular}{|c|c|c|c|c|c|c|}
\hline SNP & Substitution & $\begin{array}{l}\text { Predicted } \\
\text { Functionality }^{\text {a }}\end{array}$ & $G_{e n e}{ }^{b}$ & Gene Description & Gene Ontologyc & OMIM $^{d}$ \\
\hline rs 1052486 & S625P & Possibly damaging & BAT3 & HLA-B associated transcript 3 & protein modification & 142590 \\
\hline rs7214723 & E375G & Possibly damaging & CAMKK1 & $\begin{array}{l}\text { calcium/calmodulin- } \\
\text { dependent protein kinase } \\
\text { kinase } 1, \alpha\end{array}$ & $\begin{array}{l}\text { nucleotide binding, kinase } \\
\text { activity }\end{array}$ & \\
\hline rs560191 & D353E & & TP53BP1 & $\begin{array}{l}\text { tumor protein p53 binding } \\
\text { protein, } 1\end{array}$ & regulation of transcription & 605230 \\
\hline rs3130618 & R41L & Possibly damaging & BAT4 & HLA-B associated transcript 4 & nucleic acid binding & 142610 \\
\hline rs2602141 & K1136Q & & TP53BP1 & $\begin{array}{l}\text { tumor protein p53 binding } \\
\text { protein, } 1\end{array}$ & regulation of transcription & 605230 \\
\hline rs6964587 & M463I & Possibly damaging & AKAP9 & $\begin{array}{l}\text { A kinase (PRKA) anchor } \\
\text { protein } 9\end{array}$ & $\begin{array}{l}\text { receptor binding, signal } \\
\text { transduction }\end{array}$ & 604001 \\
\hline rs2229742 & R448G & Possibly damaging & NRIP1 & $\begin{array}{l}\text { nuclear receptor interacting } \\
\text { protein } 1\end{array}$ & $\begin{array}{l}\text { receptor signaling, regulation of } \\
\text { transcription }\end{array}$ & 602490 \\
\hline rs2660744 & Q488X & Stop codon & PPAT & $\begin{array}{l}\text { phosphoribosyl pyrophosphate } \\
\text { amidotransferase }\end{array}$ & $\begin{array}{l}\text { transferase activity, nucleoside } \\
\text { metabolism }\end{array}$ & 172450 \\
\hline rs3206824 & R335G & & DKK3 & dickkopf homolog 3 & receptor signaling & 605416 \\
\hline rs6183 & P495T & $\begin{array}{l}\text { Intolerant, Probably } \\
\text { damaging }\end{array}$ & $G H R$ & growth hormone receptor & endocytosis & 600946 \\
\hline rs1129923 & G131S & Intolerant & DUSP23 & dual specificity phosphatase 23 & $\begin{array}{l}\text { protein tyrosine/serine/ } \\
\text { threonine phosphatase } \\
\text { activity }\end{array}$ & \\
\hline rs11571833 & K3326X & Stop codon & $B R C A 2$ & breast cancer 2 , early onset & $\begin{array}{l}\text { DNA repair, regulation of } \\
\text { transcription }\end{array}$ & 600185 \\
\hline rs2242089 & V105M & Intolerant & PYCRL & $\begin{array}{l}\text { pyrroline-5-carboxylate } \\
\text { reductase-like }\end{array}$ & $\begin{array}{l}\text { oxidoreductase activity, } \\
\text { electron transport }\end{array}$ & \\
\hline rs1738023 & N215D & Possibly damaging & $A K R 7 A 3$ & $\begin{array}{l}\text { aldo-keto reductase family } 7 \text {, } \\
\text { member } A 3\end{array}$ & aldehyde metabolism & 608477 \\
\hline rs11569705 & $\mathrm{D} 22 \mathrm{Y}$ & & SULT1E1 & $\begin{array}{l}\text { sulfotransferase family } 1 \mathrm{E}, \\
\text { estrogen-preferring, } \\
\text { member } 1\end{array}$ & steroid metabolism & 600043 \\
\hline rs2295778 & P41A & Possibly damaging & HIF1AN & $\begin{array}{l}\text { hypoxia-inducible factor } 1, \alpha \\
\text { subunit inhibitor }\end{array}$ & regulation of transcription & 606615 \\
\hline rs2306022 & V433M & Intolerant & ITGA11 & integrin, $\alpha 11$ & receptor signaling & 604789 \\
\hline rs10115703 & R19W & & CER1 & $\begin{array}{l}\text { cerberus } 1 \text { homolog, cysteine } \\
\text { knot superfamily }\end{array}$ & cell signaling & 603777 \\
\hline rs2108978 & $\mathrm{R} 249 \mathrm{H}$ & Probably damaging & AKAP10 & $\begin{array}{l}\text { A kinase (PRKA) anchor protein } \\
10\end{array}$ & signal transduction & 604694 \\
\hline rs2725362 & L1074F & & WRN & Werner syndrome & DNA metabolism & 604611 \\
\hline rs3732401 & P366S & Possibly damaging & GTF2E1 & $\begin{array}{l}\text { general transcription factor IIE, } \\
\text { polypeptide } 1, \alpha 56 \mathrm{kDa}\end{array}$ & regulation of transcription & 189962 \\
\hline rs4371716 & V68M & Probably damaging & $\mathrm{CDH} 12$ & $\begin{array}{l}\text { cadherin } 12 \text {, type } 2 \text { ( } \mathrm{N} \text {-cadherin } \\
\text { 2) }\end{array}$ & cell adhesion & 600562 \\
\hline rs8065506 & $\mathrm{K} 135 \mathrm{~N}$ & & ZNF624 & zinc finger protein 624 & regulation of transcription & \\
\hline rs970547 & G1894S & & COL12A1 & collagen, type XII, $\alpha 1$ & cell adhesion & 120320 \\
\hline rs2032729 & N220S & & ZNF24 & zinc finger protein 24 (KOX 17) & regulation of transcription & 194534 \\
\hline rs2243639 & T180A & & SFTPD & $\begin{array}{l}\text { surfactant, pulmonary- } \\
\text { associated protein D }\end{array}$ & cell proliferation & 178635 \\
\hline rs17632786 & N700S & $\begin{array}{l}\text { Intolerant, Possibly } \\
\text { damaging }\end{array}$ & THBS1 & thrombospondin 1 & cell adhesion, cell motility & 188060 \\
\hline rs10787428 & E131G & $\begin{array}{l}\text { Intolerant, Possibly } \\
\text { damaging }\end{array}$ & GPAM & $\begin{array}{l}\text { glycerol-3-phosphate } \\
\text { acyltransferase, mitochondrial }\end{array}$ & lipid metabolism & 602395 \\
\hline rs17184326 & K522N & & POP1 & $\begin{array}{l}\text { processing of precursor } 1 \text {, } \\
\text { ribonuclease subunit }\end{array}$ & tRNA catabolism & 602486 \\
\hline rs11575194 & R138W & & IGFBP5 & $\begin{array}{l}\text { insulin-like growth factor } \\
\text { binding protein } 5\end{array}$ & cell growth, signal transduction & 146734 \\
\hline rs363504 & L902S & & GRIK1 & $\begin{array}{l}\text { glutamate receptor, ionotropic, } \\
\text { kainate } 1\end{array}$ & cell signaling & 138245 \\
\hline rs28360135 & I134T & & $X R C C 4$ & $\begin{array}{l}\text { X-ray repair complementing } \\
\text { defective repair in Chinese } \\
\text { hamster cells } 4\end{array}$ & $\begin{array}{l}\text { DNA repair, DNA } \\
\text { recombination }\end{array}$ & 194363 \\
\hline rs1051740 & Y113H & $\begin{array}{l}\text { Intolerant, Possibly } \\
\text { damaging }\end{array}$ & EPHX1 & $\begin{array}{l}\text { epoxide hydrolase } 1, \\
\text { microsomal }\end{array}$ & xenobiotic metabolism & 132810 \\
\hline rs16900023 & P786S & & MSH5 & mutS homolog 5 (E. coli) & DNA repair, DNA metabolism & 603382 \\
\hline rs1043261 & Q484X & Stop codon & IL17RB & interleukin 17 receptor B & cell growth & 605458 \\
\hline rs2295000 & S535L & & DATF1 & $\begin{array}{l}\text { death associated transcription } \\
\text { factor } 1\end{array}$ & $\begin{array}{l}\text { apoptosis, regulation of } \\
\text { transcription }\end{array}$ & 604140 \\
\hline rs1019670 & N150I & Intolerant & $M S 4 A 6 A$ & $\begin{array}{l}\text { membrane-spanning } \\
\text { 4-domains, subfamily A, } \\
\text { member } 6 \text { A }\end{array}$ & signal transduction & 606548 \\
\hline
\end{tabular}


Table 2. Continued

\begin{tabular}{|c|c|c|c|c|c|c|}
\hline SNP & Substitution & $\begin{array}{l}\text { Predicted } \\
\text { Functionality }\end{array}$ & Gene $^{\text {b }}$ & Gene Description & Gene Ontology ${ }^{c}$ & OMIM $^{d}$ \\
\hline rs7998427 & E117G & Possibly damaging & SETDB2 & SET domain, bifurcated 2 & $\begin{array}{l}\text { chromatin modification, } \\
\text { methyltransferase activity }\end{array}$ & 607865 \\
\hline rs9262138 & D566G & Possibly damaging & DHX16 & $\begin{array}{l}\text { DEAH (Asp-Glu-Ala-His) box } \\
\text { polypeptide } 16\end{array}$ & cell cycle control & 603405 \\
\hline rs5745549 & S914N & & MSH4 & muts homolog 4 (E. coli) & $\begin{array}{l}\text { DNA repair, meiotic } \\
\text { recombination }\end{array}$ & 602105 \\
\hline rs3107275 & P421L & Intolerant & PHACS & $\begin{array}{l}\text { 1-aminocyclopropane-1- } \\
\text { carboxylate synthase }\end{array}$ & $\begin{array}{l}\text { transferase activity, amino acid } \\
\text { metabolism }\end{array}$ & 608405 \\
\hline rs8069344 & $\mathrm{L} 782 \mathrm{H}$ & Probably damaging & GUCY2D & $\begin{array}{l}\text { guanylate cyclase 2D, } \\
\text { membrane (retina specific) }\end{array}$ & cell signaling & 600179 \\
\hline rs5388 & V136I & & GH1 & growth hormone 1 & signal transduction & 139250 \\
\hline rs1800974 & $\mathrm{R} 651 \mathrm{H}$ & & ITGA7 & integrin, $\alpha 7$ & cell signaling & 600536 \\
\hline rs1820128 & C209R & Probably damaging & ZNF600 & zinc finger protein 600 & $\begin{array}{l}\text { nucleic acid binding, zinc ion } \\
\text { binding }\end{array}$ & \\
\hline rs17246389 & L6V & Intolerant & SERPINI2 & $\begin{array}{l}\text { serine proteinase inhibitor, } \\
\text { clade I, member } 2\end{array}$ & cell motility & 605587 \\
\hline rs17337252 & M234T & Possibly damaging & RB1CC1 & RB1-inducible coiled-coil 1 & kinase activity & 606837 \\
\hline rs3758938 & $\mathrm{K} 101 \mathrm{~T}$ & $\begin{array}{l}\text { Intolerant, Possibly } \\
\text { damaging }\end{array}$ & $T B \times 10$ & T-box 10 & regulation of transcription & 604648 \\
\hline rs1800076 & R75Q & $\begin{array}{l}\text { Intolerant, Possibly } \\
\text { damaging }\end{array}$ & CFTR & $\begin{array}{l}\text { CFT conductance regulator, } \\
\text { ATP-binding (sub-family C, } \\
\text { member 7) }\end{array}$ & ion transport & 602421 \\
\hline rs2230674 & P191A & Probably damaging & ATF1 & activating transcription factor 1 & regulation of transcription & 123803 \\
\hline rs2274750 & $\mathrm{A} 1781 \mathrm{~T}$ & Intolerant & $T N C$ & tenascin C (hexabrachion) & cell adhesion & 187380 \\
\hline rs933135 & R257H & & PLCD1 & phospholipase C, $\delta 1$ & $\begin{array}{l}\text { intracellular signaling, } \\
\text { phospholipid metabolism }\end{array}$ & 602142 \\
\hline rs5743611 & R80T & Probably damaging & TLR1 & toll-like receptor 1 & $\begin{array}{l}\text { regulation of TNF- } \alpha \\
\text { biosynthesis, macrophage } \\
\text { activation }\end{array}$ & 601194 \\
\hline rs1211554 & D268Y & & HUS1B & $\begin{array}{l}\text { HUS1 checkpoint homolog b } \\
\text { (S. pombe) }\end{array}$ & cell cycle control & \\
\hline rs4647932 & P464L & Possibly damaging & FGFRL1 & $\begin{array}{l}\text { fibroblast growth factor } \\
\text { receptor-like } 1\end{array}$ & receptor activity & 605830 \\
\hline rs17356233 & $\mathrm{H} 350 \mathrm{Q}$ & Possibly damaging & CHD1L & $\begin{array}{l}\text { chromodomain helicase DNA } \\
\text { binding protein 1-like }\end{array}$ & DNA repair & \\
\hline rs3738888 & $\mathrm{R} 658 \mathrm{C}$ & & BARD1 & $\begin{array}{l}\text { BRCA1 associated RING } \\
\text { domain } 1\end{array}$ & $\begin{array}{l}\text { apoptosis, DNA damage } \\
\text { response }\end{array}$ & 601593 \\
\hline rs12500797 & E1606K & Intolerant & PTPN13 & $\begin{array}{l}\text { protein tyrosine phosphatase, } \\
\text { non-receptor type } 13\end{array}$ & $\begin{array}{l}\text { protein amino acid } \\
\text { dephosphorylation }\end{array}$ & 600267 \\
\hline rs4988492 & L75F & & GHRH & $\begin{array}{l}\text { growth hormone releasing } \\
\text { hormone }\end{array}$ & $\begin{array}{l}\text { cell signaling, signal } \\
\text { transduction }\end{array}$ & 139190 \\
\hline rs2230339 & R63Q & Intolerant & GPR68 & G protein-coupled receptor 68 & $\begin{array}{l}\text { inflammatory response, signal } \\
\text { transduction }\end{array}$ & 601404 \\
\hline rs2229424 & $\mathrm{R} 1694 \mathrm{H}$ & $\begin{array}{l}\text { Intolerant, Possibly } \\
\text { damaging }\end{array}$ & FASN & fatty acid synthase & fatty acid biosynthesis & 600212 \\
\hline rs4791641 & P367L & Probably damaging & PFAS & $\begin{array}{l}\text { phosphoribosyl- } \\
\text { formylglycinamidine } \\
\text { synthase (FGAR } \\
\text { amidotransferase) }\end{array}$ & purine nucleotide biosynthesis & 602133 \\
\hline rs11652709 & Q122H & Probably damaging & EPX & eosinophil peroxidase & oxidative stress & 131399 \\
\hline rs1801690 & W335S & Probably damaging & $\mathrm{APOH}$ & $\begin{array}{l}\text { apolipoprotein } \\
\mathrm{H}(\beta \text {-2-glycoprotein I) }\end{array}$ & cellular defense & 138700 \\
\hline
\end{tabular}

aFunctional predictions based on SIFT (Intolerant) and PolyPhen (Probably damaging, Possibly damaging).

${ }^{b} \mathrm{NCBI}$ Entrez Gene.

'Gene Ontology Database (http://www.geneontology.org).

dOnline Mendelian Inheritance in Man (http://www.ncbi.nlm.nih.gov/entrez/query.fcgi?db=OMIM).

death associated transcription factor 1 [DATF1]), or the DNA damage-response pathway (BRCA2, MSH4, MSH5, XRCC4), thereby having relevance to the pathobiology of lung cancer a priori.

There is evidence that several of the associated SNPs directly impact on the structure and function of the expressed protein, and are therefore likely to be directly responsible for the observed association. SNPs BRCA2 K3326X and THBS1 N700S are preeminent in this respect. The K3326X polymorphism in BRCA2 results in loss of the terminal 91 amino acids of the expressed protein. The C-terminal region of BRCA2 is involved in the nuclear colocalization of Fanconi anemia complementation group D2 (FANCD2) (Wang et al. 2004) and cells lacking the terminal 188 amino acids of $B R C A 2$ are hypersensitive to radiation (Morimatsu et al. 1998). SNP K3326X has been reported to play a role in BRCA2-related Fanconi's anemia (Howlett et al. 2002) and recently reported to increase the risk of pancreatic cancer (Martin et al. 2005). The N700S SNP of THBS1, encoding the anti-angiogenic protein thrombospondin, impacts on calcium binding vital for the normal function of THBS1, and has 
been established to critically affect the structure and function of the expressed protein (Stenina et al. 2005).

For 37 SNPs correlated with lung cancer risk, evidence that they are deleterious is supported by predictions of functionality based on the PolyPhen and/or SIFT programs. Although in silico predictions about the functional consequences of amino acid changes are in part speculative, such algorithms have been demonstrated in benchmarking studies to successfully categorize $80 \%$ of amino acid substitutions (Xi et al. 2004). Two of these 37 putatively deleterious substitutions, A1718T (rs2274750) and P464L (rs4647932), were located in genes Tenascin C (TNC) and fibroblast growth-factor receptor-like 1 (FGFRL1), respectively. Both of these genes have been shown to be differentially expressed in the various lung cancer histologies (Garber et al. 2001) and form part of the extended GH-IGF pathway, with FGFRL1 binding to fibroblast growth factor 2 (FGF2) and TNC interacting with epidermal growth factor receptor (EGFR) and IGFBP5.

Eleven of the 64 associated SNPs map to genes encoding pivotal components of the GH-IGF1 pathways (Fig. 1). The absence of suitable nsSNPs in AKT1, ARG2, FGF2, IGF1, PZDK1, and PRKCE did not permit us to examine whether variants in these genes also contribute to lung cancer susceptibility. The prior probability of identifying a significant association with lung cancer risk for a series of 11 SNPs mapping to a single defined pathway of genes is intuitively small. The assertion that polymorphic variation and subsequent dysregulation in the GH-IGF axis could be associated with risk of lung cancer is not without precedent. IGF1, which is up-regulated by $G H$, regulates cellular proliferation and apoptosis and has been shown to increase tumor growth (Khandwala et al. 2000). Elevated levels of circulating IGF1 have been shown to confer an increased risk of various tumors including breast (Toniolo et al. 2000), colorectal (Ma et al. 1999), lung (Yu et al. 1999), and prostate cancers (Chan et al. 1998). Furthermore, polymorphic variation in IGFBP3 has been

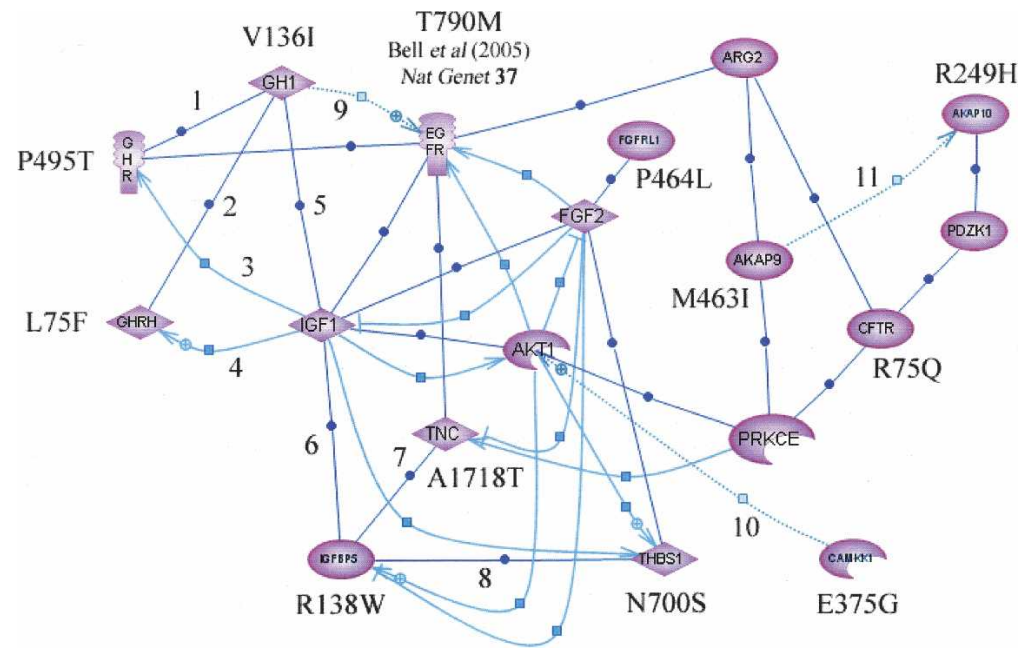

Figure 1. Inter-relationship between genes involved in the GH-IGF pathway containing SNPs associated with risk of lung cancer. Interactions were established using Pathway Assist software and are color-coded as follows: blue (expression), gray (regulation), and red (protein binding). Supporting publications are indicated with the corresponding NCBI Entrez PubMed ID in square brackets. (1) Binding [12,888,636]; (2) Binding [11,832,396]; (3) Expression [11,849,991]; (4) Expression $[11,606,442] ;(5)$ Binding $[11,126,270] ;$ (6) Binding [15,140,223]; (7) Binding $[10,982,804]$; (8) Binding $[11,751,588]$; (9) Regulation [14,517,795]; (10) Regulation [11,395,482]; (11) Regulation $[15,047,863]$. Validated nsSNPs with frequency data from Caucasian populations were not available in dbSNP Build 123 for genes AKT1, ARC2, FGF2, IGF1, PZDK1, and PRKCE. Bell et al. (2005) found association between lung cancer and SNP T790M. reported to increase risk of non-small cell lung cancer (NSCLC) (Moon et al. 2006). Recently, Bell et al. (2005) demonstrated that inherited susceptibility to lung cancer may be associated with acquisition of drug resistance mediated by EGFR T790M. While and cancer to date, it is noteworthy that IGFBP5 is required for

While it is desirable to validate our findings through analysis of additional large data sets, our study provides evidence that inherited predisposition to lung cancer is in part mediated through low-penetrance alleles and specifically identifies varialleles.

\section{Methods}

\section{Patients and control subjects}

Patients with lung cancer were ascertained from the Genetic Lung Cancer Predisposition Study (GELCAPS) based in the United Kingdom (UK). Information on clinico-pathological characteristics and family history was collected using standardized aires (Matakidou et al. 2005). In total, 1529 individuals with lung cancer were included in the study (506 males and 1023 selection was prioritized firstly by family history and secondly, by early age-at-diagnosis. A total of 573 cases (38\%) had a parent affected with lung cancer. Only 97 (6.3\%) of 1529 cases were nonsmokers. Histology information was available for 1489 the lung cancer cases-387 were small-cell cases and 1098 were non-small-cell cases (of which 483 were squamous and 343 adenocarcinomas).

A total of 2707 healthy individuals were recruited through either the Royal Marsden Hospital Trust/Institute of Cancer Research Family History and DNA Registry (1999-2004; http://intra-test.icr.ac.uk/ tissueres/patient_blood.html), the National Study of Colorectal Cancer Genetics Trial (2004; http://www.ncrn.org. uk/portfolio/data.asp?ID=1269) or GELCAPS, all established within the UK. The control group contained 836 (31\%) males and 1871 (69\%) females, median age $59 \mathrm{yr}$ (range 21-92 yr). None of the controls reported a personal history of cancer. All cases and controls were British Caucasians and there were no obvious demographic differences between groups in terms of place of residence within the UK. All study participants provided written informed consent. Ethical approval for the study was obtained from the London Multi-Center Research Ethics Committee (MREC/98/ 2/67) in accordance with the tenets of the Declaration of Helsinki. DNA was extracted from blood samples using conventional methodologies and quantified using PicoGreen (Invitrogen).

\section{Selection of candidate genes and SNPs}

We have previously established a publicly accessible PICS (Predicted Impact of 
Coding SNPs) database (http://www.icr.ac.uk/cancgen/molgen/ MolPopGen_PICS_database.htm) of potentially functional nsSNPs in genes with relevance to cancer biology (Rudd et al. 2005). Briefly, candidate cancer genes were identified by interrogating the Gene Ontology Consortium database (http://www. geneontology.org; Ashburner et al. 2000), Kyoto Encyclopedia of Genes and Genomes database (http://www.genome.jp/kegg; Kanehisa et al. 2004), Stratagene's Interaction Explorer Pathway Assist Program (http://www.iobion.com/news/hotnews. html? $\mathrm{cmd}=$ Retrieve\&dopt=Abstract), National Center for Biotechnology Information (NCBI) Entrez Gene database (http://www. ncbi.nlm.nih.gov/entrez/query.fcgi?CMD=search\&DB=gene; Maglott et al. 2005), and the CancerGene database (http:// caroll.vjf.cnrs.fr/cancergene/HOME.html). A total of 9537 validated nsSNPs with minor allele frequency (MAF) data were identified within 21,506 LocusLink annotated genes in NCBI dbSNP Build 123 (http://www.ncbi.nlm.nih.gov/SNP/; Sherry et al. 2001). Filtering this list and linking it to 7080 candidate cancer genes yielded 3666 validated nsSNPs with MAF $\geq 0.01$ in Caucasian populations. The functional impact of nsSNPs was predicted using the in silico computational tools PolyPhen (http://www. bork.embl-heidelberg.de/PolyPhen/; Ramensky et al. 2002) and SIFT (version 2.1; http://blocks.fhcrc.org/sift/SIFT.html; Ng and Henikoff 2001). Using the PICS database and published work on resequencing of DNA repair genes (Ford et al. 2000; Kuschel et al. 2002; Mohrenweiser et al. 2002; Fearnhead et al. 2004; Savas et al. 2004) we prioritized a set of 1476 nsSNPs for the current study. For those SNPs yet to be documented in the latest release of NCBI dbSNP (Build 125), we have submitted complete genotype information including MAF to NCBI and assigned the resultant dbSNP 'ss' designations accordingly. Annotated flanking sequence information for each SNP was derived from the University of California Santa Cruz (UCSC) Human Genome Browser (Assembly hg17; http://genome.ucsc.edu/cgi-bin/hgGateway).

\section{SNP genotyping and data manipulation}

Genotyping of samples was performed using customized Illumina Sentrix Bead Arrays according to the manufacturer's protocols. DNA samples with GenCall scores $<0.25$ at any locus were considered "no calls." A DNA sample was deemed to have failed if it generated genotypes at $<95 \%$ of loci. A SNP was deemed to have failed if $<95 \%$ of DNA samples generated a genotype at the locus. Conversion of genotype data into formats suitable for processing was performed using in-house Perl scripts (available upon request). Conventional statistical manipulations were undertaken in STATA (version 8; http://www.stata.com), S-Plus (version 7; http://www.insightful.com), or R (version 2.0.0; http:// www.r-project.org).

\section{Population stratification}

Genotypic frequencies in control subjects for each SNP were tested for departure from Hardy-Weinberg equilibrium (HWE) using a $\chi^{2}$ test or Fisher's exact test, where an expected cell count was less than five. SNPs that violate HWE in the control population can indicate selection bias or genotyping errors; these were removed from further analysis. To detect and control for possible population stratification, we used the genomic control approach (Devlin and Roeder 1999), using all SNPs to estimate the stratification parameter $\hat{\lambda}$ and its associated 95\% confidence interval (CI).

\section{Risk of lung cancer associated with nsSNPs}

The most efficient test of association depends on the true mode of allelic inheritance. Since this is not known, we based our analyses on the difference between allelic frequencies in cases and controls using a $\chi^{2}$ test with one degree of freedom, or Fisher's exact test if the expected numbers in individual cells were less than five. We denote this test statistic $\mathrm{T}_{\mathrm{A}}$ with corresponding $P$-value $P_{\mathrm{A}}$. We also investigated two further tests based on $2 \times 2$ tables combining the heterozygotes with either the common or rare homozygotes to derive the statistics $T_{R}$ and $T_{D}$ with corresponding $P$-values $P_{\mathrm{R}}$ and $P_{\mathrm{D}}$, which are most powerful under recessive or dominant models, respectively. The risks associated with each SNP were estimated by allelic, dominant, and recessive odds ratios (ORs) using unconditional logistic regression. Associated $95 \%$ confidence intervals (CI) were calculated in each case. Where it was not possible to calculate ORs by asymptotic methods, an exact approach was implemented using LogXact software (http://www.cytel.com; Cytel Corporation).

To increase the power to detect associations, we further analyzed case and control genotypes adopting a set-association approach, combining the largest $\mathrm{T}_{\mathrm{A}}$ statistics from individual tests into a single genome-wide statistic to model the joint effects of individual loci on lung cancer risk. Set-association analysis was conducted using the Sumstat program (Hoh et al. 2001), performing 50,000 iterations, and setting the maximum possible number of terms in the sum to be 100 . The significance of this statistic was estimated through permutation, adjusting for the number of terms in the set being, a priori, unknown.

\section{Multiple testing}

Standard approaches to adjust for multiple testing such as the Bonferroni correction are known to be conservative due to their reliance on the assumption of independence between tests, which can lead to type I errors. To control error rate, we adopted an empirical Monte Carlo simulation approach (Churchill and Doerge 1994) based on 10,000 permutations, which takes into account the fact that tests may be correlated due to the presence of LD throughout the genome. At each iteration, case and control labels are permuted at random and maximum test statistics $\mathrm{T}_{\mathrm{A}}{ }^{\max }, \mathrm{T}_{\mathrm{D}}{ }^{\text {max }}$, and $\mathrm{T}_{\mathrm{R}}{ }^{\text {max }}$ are determined. Significance levels of the observed statistics from the original data are then estimated by the proportion of permutation samples with $\mathrm{T}^{\mathrm{max}}$ larger than that in the observed data. Although this approach adjusts for multiple testing for each of the three statistics separately, the consequent increase in false-positive rate is expected to be small due to the strong dependence between tests.

\section{Assessment of linkage disequilibrium between SNPs}

To identify SNPs in high LD, we calculated the pairwise LD measure $D^{\prime}$ between consecutive pairs of markers throughout the genome using the expectation-maximization algorithm to estimate two-locus haplotype frequencies. We computed $D^{\prime}$ for SNPs with MAF $>0.1 \%$, as the distribution of LD estimates for SNPs with smaller MAF was found to be unstable. For the purposes of this study, a pair of SNPs was defined as being in high LD if they had pairwise LD measure $D^{\prime}>0.5$. This information was used to investigate the relationship between haplotypes and disease status. Specifically, haplotypes were reconstructed using a Markov chain Monte Carlo method, and their frequencies in cases and controls compared by permutation testing using the program PHASE (Stephens et al. 2001; Stephens and Donnelly 2003).

\section{Covariates and interactions}

Information on a number of covariates was available for the cases, including family history of lung cancer, histology, age at diagnosis, smoking history, and asbestos exposure. Analyses were only undertaken for subgroups with a sample size $>300$. The test 
statistics $T_{A}, T_{R}$, and $T_{D}$ were computed for all subgroups, together with ORs and their associated 95\% CIs. The set association approach was also implemented for each subgroup.

Under certain conditions, a two-stage process incorporating estimates of pairwise interaction between significant SNPs can yield greater power to detect association (Marchini et al. 2005). To investigate epistatic interactions, each pair of SNPs that showed significant allelic association at the 5\% level were tested fitting a saturated logistic regression model, and the log likelihood ratio statistic for comparison with the main effects model computed. This was compared against a $\chi^{2}$ distribution with 1 d.f. Statistics were then adjusted for multiple testing using a Bonferroni correction.

\section{Acknowledgments}

Funding for this work was undertaken with support from Cancer Research UK, the Arbib Foundation, HEAL, the National Cancer Research Network, the European Union Network of Excellence, and the Institute of Cancer Research. A.M. was the recipient of a clinical research fellowship from the Allan J. Lerner Fund. We gratefully acknowledge the participation of patient and control individuals. The authors are indebted to Richard Coleman, Christina Fleischmann, Olivia Fletcher, Nick Hearle, Nichola Johnson, Rosalind Mutch, Claire Palle, Julian Peto, Mobshra Qureshi, Elaine Ryder-Mills, Hayley Spendlove, and Remben Talaban for sample ascertainment. We thank Jurg Ott for access to a recompiled version of his Sumstat program.

\section{References}

Ashburner, M., Ball, C.A., Blake, J.A., Botstein, D., Butler, H., Cherry, J.M., Davis, A.P., Dolinski, K., Dwight, S.S., Eppig, J.T., et al. 2000. Gene ontology: Tool for the unification of biology. The Gene Ontology Consortium. Nat. Genet. 25: 25-29.

Bailey-Wilson, J.E., Amos, C.I., Pinney, S.M., Petersen, G.M., de Andrade, M., Wiest, J.S., Fain, P., Schwartz, A.G., You, M., Franklin, W., et al. 2004. A major lung cancer susceptibility locus maps to chromosome 6q23-25. Am. J. Hum. Genet. 75: 460-474.

Bell, D.W., Gore, I., Okimoto, R.A., Godin-Heymann, N., Sordella, R., Mulloy, R., Sharma, S.V., Brannigan, B.W., Mohapatra, G. Settleman, J., et al. 2005. Inherited susceptibility to lung cancer may be associated with the T790M drug resistance mutation in EGFR. Nat. Genet. 37: 1315-1316.

Chan, J.M., Stampfer, M.J., Giovannucci, E., Gann, P.H., Ma, J., Wilkinson, P., Hennekens, C.H., and Pollak, M. 1998. Plasma insulin-like growth factor-I and prostate cancer risk: A prospective study. Science 279: 563-566.

Churchill, G.A. and Doerge, R.W. 1994. Empirical threshold values for quantitative trait mapping. Genetics 138: 963-971.

Devlin, B. and Roeder, K. 1999. Genomic control for association studies. Biometrics 55: 997-1004.

Doll, R. and Peto, R. 1981. The causes of cancer: Quantitative estimates of avoidable risks of cancer in the United States today. J. Natl. Cancer Inst. 66: 1191-1308.

Fearnhead, N.S., Wilding, J.L., Winney, B., Tonks, S., Bartlett, S., Bicknell, D.C., Tomlinson, I.P., Mortensen, N.J., and Bodmer, W.F. 2004. Multiple rare variants in different genes account for multifactorial inherited susceptibility to colorectal adenomas. Proc. Natl. Acad. Sci. 101: 15992-15997.

Ford, B.N., Ruttan, C.C., Kyle, V.L., Brackley, M.E., and Glickman, B.W 2000. Identification of single nucleotide polymorphisms in human DNA repair genes. Carcinogenesis 21: 1977-1981.

Garber, M.E., Troyanskaya, O.G., Schluens, K., Petersen, S., Thaesler, Z., Pacyna-Gengelbach, M., van de Rijn, M., Rosen, G.D., Perou, C.M., Whyte, R.I., et al. 2001. Diversity of gene expression in adenocarcinoma of the lung. Proc. Natl. Acad. Sci. 98: 13784-13789.

Hoh, J., Wille, A., and Ott, J. 2001. Trimming, weighting, and grouping SNPs in human case-control association studies. Genome Res. 11: $2115-2119$.

Howlett, N.G., Taniguchi, T., Olson, S., Cox, B., Waisfisz, Q., De Die-Smulders, C., Persky, N., Grompe, M., Joenje, H., Pals, G., et al.
2002. Biallelic inactivation of BRCA2 in Fanconi anemia. Science 297: 606-609.

Hwang, S.J., Cheng, L.S., Lozano, G., Amos, C.I., Gu, X., and Strong, L.C. 2003. Lung cancer risk in germline p53 mutation carriers: Association between an inherited cancer predisposition, cigarette smoking, and cancer risk. Hum. Genet. 113: 238-243.

IARC (International Agency for Research on Cancer). 1986. IARC Monographs on the evaluation of the carcinogenic risk of chemicals to humans: Tobacco smoking. IARC, Lyon, France.

Kanehisa, M., Goto, S., Kawashima, S., Okuno, Y., and Hattori, M. 2004. The KEGG resource for deciphering the genome. Nucleic Acids Res. 32: D277-D280.

Khandwala, H.M., McCutcheon, I.E., Flyvbjerg, A., and Friend, K.E. 2000. The effects of insulin-like growth factors on tumorigenesis and neoplastic growth. Endocr. Rev. 21: 215-244.

Kuschel, B., Auranen, A., McBride, S., Novik, K.L., Antoniou, A., Lipscombe, J.M., Day, N.E., Easton, D.F., Ponder, B.A., Pharoah, P.D., et al. 2002. Variants in DNA double-strand break repair genes and breast cancer susceptibility. Hum. Mol. Genet. 11: 1399-1407.

Ma, J., Pollak, M.N., Giovannucci, E., Chan, J.M., Tao, Y., Hennekens, C.H., and Stampfer, M.J. 1999. Prospective study of colorectal cancer risk in men and plasma levels of insulin-like growth factor (IGF)-I and IGF-binding protein-3. J. Natl. Cancer Inst. 91: 620-625.

Maglott, D., Ostell, J., Pruitt, K.D., and Tatusova, T. 2005. Entrez Gene: Gene-centered information at NCBI. Nucleic Acids Res. 33: D54-D58.

Marchini, J., Donnelly, P., and Cardon, L.R. 2005. Genome-wide strategies for detecting multiple loci that influence complex diseases. Nat. Genet. 37: 413-417.

Martin, S.T., Matsubayashi, H., Rogers, C.D., Philips, J., Couch, F.J. Brune, K., Yeo, C.J., Kern, S.E., Hruban, R.H., and Goggins, M. 2005. Increased prevalence of the BRCA2 polymorphic stop codon K3326X among individuals with familial pancreatic cancer. Oncogene 24: 3652-3656.

Matakidou, A., Eisen, T., and Houlston, R.S. 2005. Systematic review of the relationship between family history and lung cancer risk. $\mathrm{Br}$. $J$. Cancer 93: 825-833.

Mohrenweiser, H.W., Xi, T., Vazquez-Matias, J., and Jones, I.M. 2002. Identification of 127 amino acid substitution variants in screening 37 DNA repair genes in humans. Cancer Epidemiol. Biomarkers Prev. 11: $1054-1064$.

Moon, J.W., Chang, Y.S., Ahn, C.W., Yoo, K.N., Shin, J.H., Kong, J.H., Kim, Y.S., Chang, J., Kim, S.K., Kim, H.J., et al. 2006. Promoter-202 $\mathrm{A} / \mathrm{C}$ polymorphism of insulin-like growth factor binding protein-3 gene and non-small cell lung cancer risk. Int. J. Cancer 118: 353-356.

Morimatsu, M., Donoho, G., and Hasty, P. 1998. Cells deleted for Brca2 $\mathrm{COOH}$ terminus exhibit hypersensitivity to $\gamma$-radiation and premature senescence. Cancer Res. 58: 3441-3447.

$\mathrm{Ng}$, P.C. and Henikoff, S. 2001. Predicting deleterious amino acid substitutions. Genome Res. 11: 863-874.

Parkin, D.M., Bray, F., Ferlay, J., and Pisani, P. 2005. Global cancer statistics, 2002. CA Cancer J. Clin. 55: 74-108.

Ramensky, V., Bork, P., and Sunyaev, S. 2002. Human non-synonymous SNPs: Server and survey. Nucleic Acids Res. 30: 3894-3900.

Rudd, M.F., Williams, R.D., Webb, E.L., Schmidt, S., Sellick, G.S., and Houlston, R.S. 2005. The predicted impact of coding single nucleotide polymorphisms database. Cancer Epidemiol. Biomarkers Prev. 14: 2598-2604.

Sanders, B.M., Jay, M., Draper, G.J., and Roberts, E.M. 1989. Non-ocular cancer in relatives of retinoblastoma patients. Br. J. Cancer 60: $358-365$.

Savas, S., Kim, D.Y., Ahmad, M.F., Shariff, M., and Ozcelik, H. 2004. Identifying functional genetic variants in DNA repair pathway using protein conservation analysis. Cancer Epidemiol. Biomarkers Prev. 13: 801-807.

Schuller, A.G., van Neck, J.W., Beukenholdt, R.W., Zwarthoff, E.C., and Drop, S.L. 1995. IGF, type I IGF receptor and IGF-binding protein mRNA expression in the developing mouse lung. J. Mol. Endocrinol. 14: 349-355.

Sherry, S.T., Ward, M.H., Kholodov, M., Baker, L., Phan, L., Smigielski, E.M., and Sirokin, K. 2001. dSNP: The NCBI database of genetic variation. Nucleic Acids Res. 29: 308-311.

Stenina, O.I., Ustinov, V., Krukovets, I., Marinic, T., Topol, E.J., and Plow, E.F. 2005. Polymorphisms A387P in thrombospondin-4 and N700S in thrombospondin-1 perturb calcium binding sites. FASEB J. 19: 1893-1895.

Stephens, M. and Donnelly, P. 2003. A comparison of bayesian methods for haplotype reconstruction from population genotype data. Am. J. Hum. Genet. 73: 1162-1169.

Stephens, M., Smith, N.J., and Donnelly, P. 2001. A new statistical method for haplotype reconstruction from population data. Am. J. Hum. Genet. 68: 978-989. 
Functional nsSNPs and lung cancer risk

Takemiya, M., Shiraishi, S., Teramoto, T., and Miki, Y. 1987. Bloom's syndrome with porokeratosis of Mibelli and multiple cancers of the skin, lung and colon. Clin. Genet. 31: 35-44.

Toniolo, P., Bruning, P.F., Akhmedkhanov, A., Bonfrer, J.M., Koenig, K.L., Lukanova, A., Shore, R.E., and Zeleniuch-Jacquotte, A. 2000. Serum insulin-like growth factor-I and breast cancer. Int. J. Cancer 88: 828-832.

Wang, X., Andreassen, P.R., and D'Andrea, A.D. 2004. Functional interaction of monoubiquitinated FANCD2 and BRCA2/FANCD1 in chromatin. Mol. Cell. Biol. 24: 5850-5862.

Xi, T., Jones, I.M., and Mohrenweiser, H.W. 2004. Many amino acid substitution variants identified in DNA repair genes during human population screenings are predicted to impact protein function. Genomics 83: 970-979.

Yamanaka, A., Hirai, T., Ohtake, Y., and Kitagawa, M. 1997. Lung cancer associated with Werner's syndrome: A case report and review of the literature. Jpn. J. Clin. Oncol. 27: 415-418.

Yu, H., Spitz, M.R., Mistry, J., Gu, J., Hong, W.K., and Wu, X. 1999. Plasma levels of insulin-like growth factor-I and lung cancer risk: A case-control analysis. J. Natl. Cancer Inst. 91: 151-156.

Received January 6, 2006; accepted in revised form March 20, 2006. 


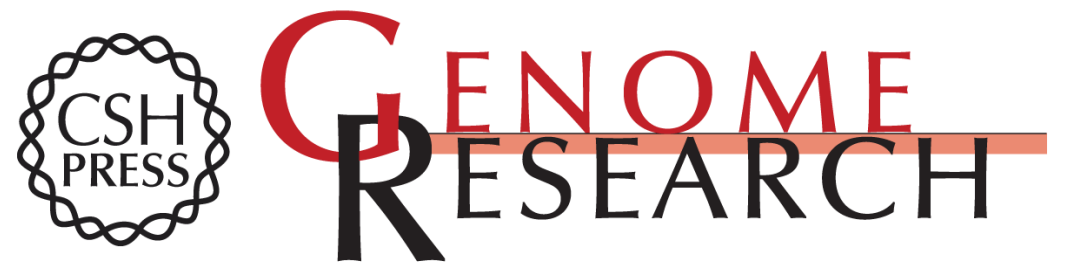

\section{Variants in the GH-IGF axis confer susceptibilityto lung cancer}

Matthew F. Rudd, Emily L. Webb, Athena Matakidou, et al.

Genome Res. 2006 16: 693-701

Access the most recent version at doi:10.1101/gr.5120106

\section{Supplemental http://genome.cshlp.org/content/suppl/2006/05/16/16.6.693.DC1 \\ Material}

References This article cites 41 articles, 13 of which can be accessed free at:

http://genome.cshlp.org/content/16/6/693.full.html\#ref-list-1

\section{License}

Email Alerting Receive free email alerts when new articles cite this article - sign up in the box at the Service top right corner of the article or click here.

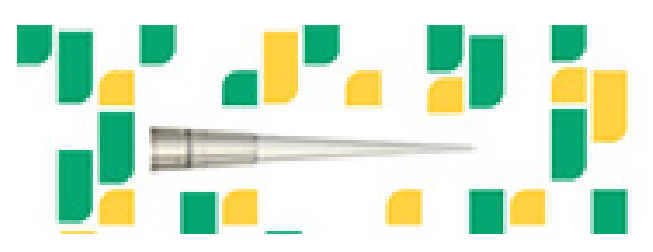

To subscribe to Genome Research go to: https://genome.cshlp.org/subscriptions 